\title{
Importance of risk assessment in the management of radioactively contaminated areas
}

\author{
B. Cessac, A. Oudiz, J. Brenot, J.P. Maigne, P. Santucci, M.-C. Robe and P. Charbonneau \\ Institut de Portection et de Sûreté Nucléaire, BP. 6, 92265 Fontenay-aux-Roses cedex, France
}

\begin{abstract}
At the request of the French ministries of Health and Environment, the IPSN has realized a methodological guide for aiding to the management of radioactively contaminated sites. This guide gives an operational framework to all stakeholders concerned by the management of such sites. It deals with industrial sites to which current nuclear regulation do not apply. The methodology consists of six stages going from the site characterization by radioactivity measurements to the definition of remediation strategies. Between them, the risk assessment is a stage of particular importance which takes place in two steps according to the importance of the situation to be treated. Firstly, a simplified risk assessment (SRA) supplies a dosimetric overview of the site based on individual dose calculations for generic scenarios of use (residential area, school, offices,...). Secondly, a detailed risk assessment (DRA) is needed when the SRA shows a level of dose judged too important for the potentially possible uses for the site. The DRA needs a finer characterization of the site and the exposure situation. In every case, the methodology applied should be optimised and imply the various stakeholders in a process of information and dialogue.
\end{abstract}

\section{CONTEXT}

In the context of the transposition into French regulation of the European Directive 96/29 concerning the safety standards for radiological exposure of population and workers [1], the French Institute for Protection and Nuclear Safety (IPSN) has been assigned by the ministries of Health and Environment the task of preparing a guide for aiding to the management of sites potentially contaminated by radioactive substances [2].

The purpose of this guide is to describe the procedures to be followed by the technical bodies responsible for assessing a site from the radiological point of view and for proposing measures. These procedures are also intended to provide a technical framework to which the government departments concerned may refer. More generally, the guide is intended to be a reference source for all stakeholders, enabling them to discuss matters on a common basis. The guide is therefore addressed to all those with a role to play at potentially contaminated sites (government departments, regional and local authorities, consultancy firms, insurance companies, operators, various associations, etc.) and who are involved in preparing, implementing or monitoring site management studies.

\section{THE METHODOLOGY}

\subsection{A graduated approach}

The guide presents an approach involving six different stages that enable the risks associated to the potential contamination of a site to be assessed and the appropriate measures to be identified in the case of a need in remediation. A distinction is made between :

The removal of doubt is based on a rapid inspection of the site with measurements of radioactivity in the areas (buildings or soils) that are assumed to be contaminated : gamma dose rate monitoring or specific alpha, beta or X-ray measurements, where necessary. 
The objective of the pre-diagnosis is to identify the real or potential risks so as to take emergency protection actions required to insure the protection of the population and workers that may be exposed to radiations due to the contamination at the site or in its vicinity.

The initial diagnosis consists in establishing a site status report in term of past activities (historical analysis), vulnerability of the environment (soil, groundwater, surface water, air) and radiological characterisation (mapping of the surface and initial subsurface studies, measurements of radioactivity in water, crops and animals that may enter the human food chain.

The next stage uses the data collected previously in order to evaluate the potential risk associated with the use of the site by the population or workers on-site or in its vicinity. The risk assessment is divided into two different steps, The simplified risk assessment (SRA) and the detailed risk assessment (DRA) described in the section 3 below.

The final stage is the assistance in selecting the remediation strategy for a given use. To help to the selection of an appropriate strategy, the principle of optimising radiation protection should be applied. Optimisation involves comparing various strategies by looking at a number of criteria (dosimetric, economic, etc.), after which the most appropriate solution can be identified.

The remediation strategy chosen depends on the future use of the site. A change in use mas sometimes facilitate clean-up or even obviate the need for it. In most cases, however, the use of clean-lip techniques has to be considered, which means that the various techniques available (removal of soli. containment in situ, installation of barriers, etc.) need to be assessed. These techniques should be looked at in conjunction with the various methods of stcring any waste that arises, in order to determine possible remediation strategies. The latter should then be characterised by taking account of the following aspects: reduction in radiological impact (doses averted, contamination avoided, doses to workers), costs, associated nuisances, durability of remediation techniques, reversibility of techniques, and the need for institutional monitoring and maintenance of the site.

A summary of the preceding characteristics, clearly identifying the uncertainties in the assessment, must be presented so that the strategies can be compared.

\subsection{The decision-making framework}

The assessment effort is often very costly and time-consuming, so it may not be necessary to implement all the stages. A decision-making framework has to be adopted at each stage in order that the assessment could be stopped, depending on circumstances. For example, "removing of doubt" could constitute the final stage, either because no contamination has been found on the site or because, at this stage, the site can readily be decontaminated. Similarly, in certain "simple" cases, remediation of the site can be decided at the pre-diagnosis, initial diagnosis or simplified risk assessment stage.

However, even if all the stages are to be completed, the effort devoted to each of them should be commensurate with the level of radiation risk and take into account the economic, social and cultural context of the site concerned.

\section{RISK ASSESSMENT IN THE WHOLE METHODOLOGY}

\subsection{Simplified risk assessment}

The simplified risk assessment (SRA) relies on the data collected during the initial diagnosis. In the guide, a distinguish is made between the contamination of the soils and the buildings. The SRA involves a calculation of the potential dose impact associated with various scenarios for the use of the site and buildings, based on the results of radioactivity measurements of the soil and the buildings at the site. To facilitate this calculation, generic scenarios have been prepared and then evaluated using a calculation model [3]. This model determines the individual effective dose (in mSv/year) associated with soll contamination that is incurred per unit of specific activity $(1 \mathrm{~Bq} / \mathrm{g}$ of soil), for the radionuclides likely to be found at contaminated sites. The generic scenarios incorporate simple assumptions that prudentially over-estimate the dose impact. 


\subsubsection{Generic scenarios considered in the guide}

\subsubsection{For use of buildings}

Two scenarios are considered for buildings :

- Buildings used for work purposes : the reference group is represented by people working in the contaminated area.

- Buildings for private use: the members of the reference group considered here are a parent who stays at home and his or her five-year-old child.

In both cases, the individuals are assumed to be exposed for the period of their stay and also during improvements in the buildings (redecorating, for example) which may put some radioactivity in suspension in the air.

\subsubsection{For use of soils}

Different scenarios are considered in the guide :

- Staying at home and playing in garden: a family spends most of its time in a single-family house, with an ordinary garden and a kitchen garden, situated on the contaminated site. The members of the reference group exhibiting the most extreme behaviour from the radiation exposure point of view are a parent who stays at home and, in particular, his or her five-year-old child.

- Working in offices: service-sector staff work regularly in offices situated above contaminated ground.

- Market garden: it is assumed that part or all of the site is planted by a market gardener for the production of vegetables (mainly lettuce, cabbage, carrots and tomatoes). It is also assumed that all the vegetables which the market gardener consumes come from his market garden and that he does not live on the site.

- Primary school: a primary school receiving children aged 5 to 10 is situated on the site. It consists of various buildings (classrooms, canteen, etc.) and a playground with a hard-packed surface. The children constitute the most sensitive group.

- Public car park: the contaminated site, which covers a small area, is converted into an open-air, unsupervised public car park. The representative individuals belonging to the reference group are drivers who park their vehicles.

Two additional scenarios have been examined to assess the radiological impact associated with two transient situations.

- Incursions onto waste land: the site is supervised so as to prevent any structures such as dwellings from being built there but children, for example, could go there to play. The reference group then consists of ten-year-old children.

- Construction site: a construction site covering the contaminated area is set up. Two situations are looked at: the construction of a car park and the construction of a building. In both cases, the reference group consists of those working at the construction site. 


\subsubsection{Dose calculation}

The dose calculation relies on the relevant data measured at the site. For buildings, at this stage there may only be a fixed surface contamination of walls and equipment. Two kinds of measurements can be used for the dose calculations : dose rates in the buildings or measured fixed activities on the walls or on irremovable equipments.

In the case of contamination of a soil, the doses may be calculated on the basis of the knowledge of the maximum dose rate above the contaminated area or of the specific activity of each radionuclide present in this soil.

In order to be as operational as possible and to help the expert in charge of the assessment the guide provides, for each generic scenario considered, different results in terms of dose calculation per unit specific activity with the associated dose rates and also some specific activities and dose rates calculated for a dose of $1 \mathrm{mSv} /$ year.

The final step of the assessment is then the comparison of the calculated dose for one or several specific scenario(s) of use to a reference level of dose, "the selection level", in order to select a potential use satisfying to the safety standards for population and workers.

\subsubsection{Setting of selection level (SL) by public authorities}

The selection level (SL) is "the individual dose (mSv/year) above which the need for remediation must be considered", the individual dose being the average dose received within the reference group. In some cases, employing a selection level obviates the need to carry out a detailed risk assessment or to examine remediation techniques, their cost and effectiveness, provided the resulting information will not lead to any substantial increase in the knowledge on which the choice made at the SRA stage is based.

The public authorities should set this level. To give a rough idea, it could be of the order of a fraction of $1 \mathrm{mSv} /$ year.

\subsubsection{Decision-making}

The simplified risk assessment (SRA) enables an initial judgement to be made of the contaminated site's radiological impact in relation with the various uses that the stakeholders have in mind for it (maintaining the current use or selecting a different future use).

The SRA enables stakeholders to identify two types of site:

- Sites that can be reused for "non-sensitive" purposes (as a car park, for example), where it is not essential to undertake a clean-up. This is the case when the doses associated with the contamination measured at the site and in the buildings remain below the "selection level". Using a selection level means that, in some cases, it is not necessary to go through the subsequent stages (a detailed risk assessment and a study of clean-up techniques, their cost and effectiveness) because the information obtained from the subsequent stages would not substantially improve the knowledge underlying a decision made at the SRA stage.

Restrictions on a future change in use will then have to be imposed. If necessary, periodic monitoring of contamination at the site and in the surrounding area will have to be arranged.

The stakeholders may decide to reduce doses if the available remediation methods mean this can be done at an acceptable economic cost. 
- Sites at which doses associated with the anticipated use exceed the selection level or sites where doses do not necessarily exceed the selection level but whose anticipated use is regarded as more "sensitive". In such a case, the stakeholders will have to carry out a detailed risk assessment before making any decision.

At a "small" site, stakeholders can arrange for the contaminated soil to be removed if it results in doses falling well below the selection level, provided this does not cause any great administrative or financial problems. After clean-up, the site can then be used without restriction.

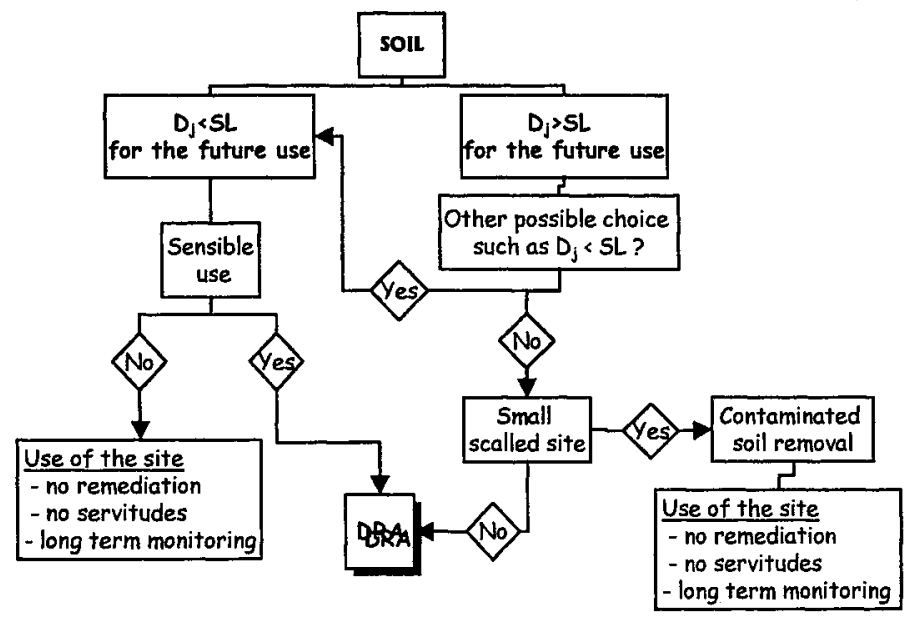

Figure 1 : Decision options following simplified risk assessment for soil

\subsection{Detailed risk assessment}

The detailed risk assessment (DRA) relates only to the soil, because any clean-up of the buildings will have taken place at an earlier stage. It involves, first of all, in-depth sampling of the site, focusing on subsurface mapping, which was deliberately cursory during the initial diagnostic stage. The detailed risk assessment also identifies the hydrogeological characteristics of the site and its environment. The envisaged future use must be specified by carrying out, if necessary, a site analysis to provide the most realistic model possible for the subsequent utilisation of the site.

The radiological impact is determined by using several indicators, depending on the specific situation:

- the individual dose to the so-called "reference group" (the most exposed) and the numbers involved;

- the average dose at the site and the numbers involved;

- the average dose at contaminated zones in the vicinity of the site and the numbers involved;

- the specific activity in the soil, in edible agricultural and woodland products, in the meat of farm animals, in milk, etc.

\subsubsection{Decision-making}

The detailed risk assessment (DRA) can be used to refine the hypotheses and select realistic data for the desired use. It helps to reduce the uncertainties inherent in the SRA and sometimes enables stakeholders to conclude that, where the use is not very "sensitive", the site be used without remediation. However, some restrictions may be attached only to subsequent changes in use combined with periodic monitoring 
of contamination at the site and in the surrounding area. If this is not the case, it is necessary to proceed to the stage of assistance in selecting a remediation strategy.

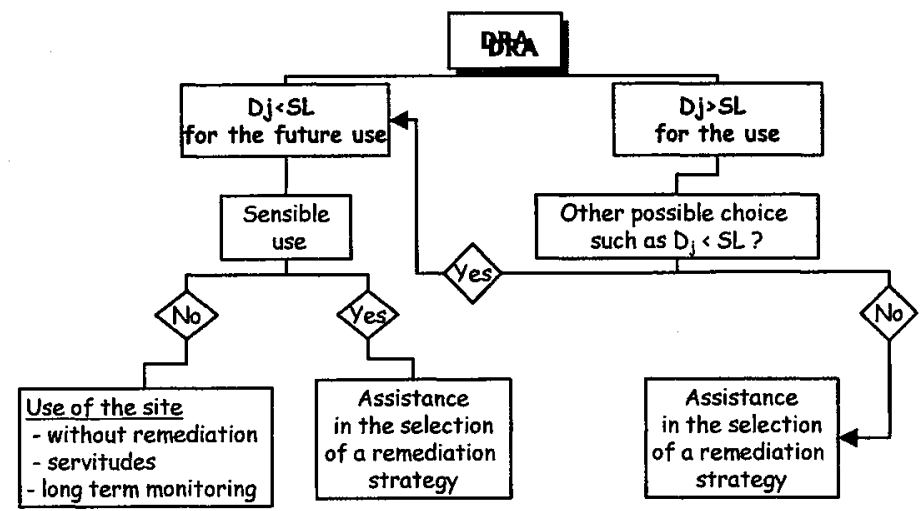

Figure 2 : Decision options following detailed risk assessment

\section{CONCLUSION}

In "simple" cases, i.e. small sites whose clean-up does not pose any technical, administrative or financial problems, risk management mainly depends on the available technical expertise. Doses and contamination of the soil and buildings have first to be assessed and then the clean-up method selected to reduce the radiological impact at an economically acceptable cost.

However, the management of contaminated sites may sometimes be much more complex, in particular because the sites are large and clean-up operations appear to be difficult and costly. For these "complex" sites, management is governed by numerous considerations, among which the radiation risk plays an important but not necessarily central role. In such cases, the remediation solution preferred by stakeholders is the result of a compromise involving various factors relating to a specific set of circumstances. However, these factors can only be taken into account credibly if the stakeholders are involved in an appropriate consultation process.

\section{References}

[1] Directive 96/29 Euratom of 13 May 1996 laying down the basic safety standards for the protection of the health of the workers and the general public against the dangers arising from ionising radiation, Commission of the European Community, JOCE $\mathrm{n}^{\circ} \mathrm{L} 159$

[2] Oudiz A., Cessac B., Brenot J., Maigne J.P. et al, Gestion des sites industriels potentiellement contaminés par des substances radioactives. Guide méthodologique. Document IPSN réalisé sous l'égide des ministères de l'Environnement et de la Santé.

[3] Cessac B., Code for individual radiation assessment for scenarios in work field and the environment. technical annexes updated 1996. Report IPSN/DPRE/SERGD N 96/10 (in French) 